\title{
Isothermal Evaporation Rate of Deposited Liquid Aerosols and the SARS-CoV-2 Coronavirus Survival
}

\section{Aerosol and Air Quality Research}

Special Issue:

Special Issue on COVID-19 Aerosol Drivers, Impacts and Mitigation (XI)

\author{
Pavel S. Grinchuk ${ }^{1 *}$, Katya I. Fisenko ${ }^{2}$, Sergey P. Fisenko ${ }^{1}$, \\ Svetlana M. Danilova-Tretiak ${ }^{1}$
}

${ }^{1}$ A.V. Luikov Heat and Mass Transfer Institute, National Academy of Sciences of Belarus, Minsk 220072, Belarus

2 Faculty of Medicine, Ludwig Maximilian University of Munich, Munich 80336, Germany

\section{OPEN ACCESS}

Received: July 22, 2020

Revised: November 4, 2020

Accepted: November 4, 2020

${ }^{*}$ Corresponding Author: gps@hmti.ac.by

\section{Publisher:}

Taiwan Association for Aerosol Research

ISSN: $1680-8584$ print

ISSN: 2071-1409 online

(c) Copyright: The Author(s). This is an open access article distributed under the terms of the Creative Commons Attribution License (CC BY 4.0), which permits unrestricted use, distribution, and reproduction in any medium, provided the original author and source are cited.

\section{ABSTRACT}

It is shown that the evaporation rate of a liquid sample with the height is about several millimeters containing the culture of coronavirus affects its survival on a substrate. Possible mechanisms of such influence can be due to the appearance of large, about 140 bar, non-comprehensive capillary pressures and the associated dynamic forces during the movement of the evaporation front in an aerosol with the virus. A simulation of isothermal evaporation of a thin liquid sample based on the Stefan problem was performed. Evaporation time of the same aqueous sample is practically twice shorter for stainless substrate than for plastic one. The comparison of simulation data and recent experiments on the coronavirus survival on various surfaces showed that the rate of isothermal evaporation of aqueous samples, which is higher for heat-conducting materials, correlates well with the lifetime of the coronavirus on these surfaces. Ceteris paribus, the viral culture should die faster on more thermally conductive and thinner substrates, as well as in lower relative humidity environment, which provide a higher evaporation rate.

Keywords: SARS-CoV-2 coronavirus, COVID-19, Vitality of coronavirus, Droplet evaporation, Substrate, Thermal conductivity

\section{INTRODUCTION}

In a recent work the experimental medical data on the survival of coronavirus on various surfaces have been presented (Doremalen et al., 2020). Deposition of liquid samples consisting of water with the coronavirus culture on a surface of various materials is common in everyday life and medicine (plastic, cardboard, stainless steel). Surprisingly the lifetime of the virus depends significantly on the substrate material and can differ by almost an order of magnitude. For brevity, below we will call these samples aqueous ones, although the composition of the liquid fraction is more complex. The mechanisms of influence of a substrate material on virus survival are not known yet. Probably it is impossible to explain such behaviour only by the specific biological interaction of viruses and materials. All experiments were carried out in laboratory conditions at a constant temperature and, apparently, constant illumination (Doremalen et al., 2020). We suppose that the evaporation rate of aqueous samples plays an important role in the survival of the coronavirus under given conditions.

The influence of such factors as temperature and humidity on the distribution of coronavirus is undeniable. The highest number of cases of SARS-CoV-2 coronavirus is observed in countries located from 30 to 50 degrees north latitude (Boulos and Geraghty, 2020). Obviously, these countries are primarily linked by the climatic factor in the sense of moderate temperatures and high humidity in winter and spring. Therefore, the evaporation rate of water samples with the virus is quite low at such conditions.

The resistance of viruses to adverse environmental factors is determined by their structure. In biology, it is customary to distinguish between simple and complex viruses. Simple or non- 
enveloped, viruses consist of nucleic acid and protein coat (capsid). Complex, or enveloped viruses are also surrounded by a lipoprotein envelope (supercapsid) outside the capsid, which makes them more vulnerable to adverse environmental factors (Cui et al., 2019). The coronavirus SARS-CoV-2 is complexly organized, has a lipoprotein membrane, and its size reaches $80-160 \mathrm{~nm}$ (Cui et al., 2019; Li et al., 2020). The characteristic size of the coronavirus protein whiskers is only 10-20 nm (Wu et al., 2020). Note that it has already been established that the coronavirus protective membrane is well wetted by water (Wu et al., 2020).

It is known from previous studies (Shaman and Kohn, 2009; Yang et al., 2012) that both absolute $(\mathrm{AH})$ and relative humidity $(\mathrm{RH})$ affect various types of viruses. Currently a number of biological and chemical mechanisms of the influence of composition of aqueous samples on viruses have been considered: water activity, surface inactivation, salt toxicity (Yang et al., 2012). We would like to discuss a purely physical mechanism of such influence here. We propose a new hypothesis about the influence of evaporation rate of aqueous samples with coronaviruses on the survival of coronaviruses. We suppose that the movement of the evaporation front through the liquid layer with the virus leads to the cessation of its activity. The resulting local nanoscale curvatures of the liquid surface lead to the appearance of huge local gradients of hydrostatic pressure, which cause damage to the virus. The high resistance of viruses to external comprehensive mechanical loads is known from previous studies (Zandi and Reguera, 2005; Aggarwal, 2018). We assumed that the lifetime of the virus during the movement of the evaporation front is significantly reduced due to additional mechanical stresses on some whiskers in a nanolayer of liquid above the virus. Indeed, when the evaporation front moves under the action of capillary forces (Laplace pressure), a pressure drop of about 10 bar at a diameter of $80 \mathrm{~nm}$ acts on the coronavirus. At the same time, a pressure of out 140 bar is already acting on his "whiskers", which have characteristic dimensions of 10-20 nm. Inhomogeneous dynamic capillary pressure forces can cause mechanical damage to the virus. Decreasing of concentration of viable viruses during evaporation of aqueous sample on a solid substrate confirms proposed physical mechanism of substrate influence (Doremalen et al., 2020).

There is at least a qualitative analogy, confirmed by different experimental data. Significant deformations of a graphene oxide sheet were experimentally discovered in an evaporating micron size droplet of water (Wang et al., 2012). Graphene is a very strong material, the elastic modulus of which reaches 1 TPa (Frank et al., 2007; Lee et al., 2008).

In any case, the presence of a virus near the evaporation front violates the symmetry of the evaporation front, which should lead to nanoscale deformations. At a higher evaporation rate, for example, for a copper substrate, deformations near the evaporation front should increase in amplitude and, as experimental data show (Doremalen et al., 2020), individual mechanical properties of each virus type begin to reveal themselves. At relatively slow evaporation rates, the difference between kinds of viruses disappears (experiments with aerosols on plastic and stainless-steel substrates with SARS-CoV-2 and SARS-CoV-1).

The purpose of this work is to show, at least semi-qualitatively, the impact of the thermal conductivity of a substrate material on the evaporation of a liquid sample with virus culture.

Thus, we connect the problem of the virus survival with the problem of evaporation of a liquid sample as we consider the conditions of experiments (Doremalen et al., 2020). The experiments were carried out at room temperature $21-23^{\circ} \mathrm{C}$. The relative humidity during the preparation of the liquid substance with the virus culture was $40 \%$. The survival rate of the coronavirus culture was evaluated for polypropylene, stainless steel from AISI 304 alloy, copper and cardboard. Thus, various domestic and hospital situations were experimentally modelled. Note that the pieces of materials were of arbitrary sizes and thicknesses. The initial concentration of the virus in the biomaterial was $10^{3.4}-10^{3.7}$ or $2500-5000 \mathrm{~mL}^{-1}$. This corresponded to the typical concentrations observed in the upper and lower respiratory tracts of a human. Next, a liquid biomaterial with a viral culture in a volume of $\mathrm{V}_{d}=50 \mu \mathrm{L}$ was applied to the surfaces described above. At certain time intervals, smears were taken from the surfaces and the virus concentration was analysed. The virus was considered deactivated at that moment in time when its concentration decreased to a value of units per millilitre. Thus, it was experimentally measured, that the lifetime of the virus was approximately 50 hours on a plastic substrate, 24 hours on a cardboard substrate, 30 hours on a stainless-steel substrate, and 5 hours on a copper substrate (Doremalen et al., 2020). These results are of great practical importance for epidemiologists in order to reach the effective prevention of the spread of coronavirus (Alvarez-Zuzek et al., 2019). 

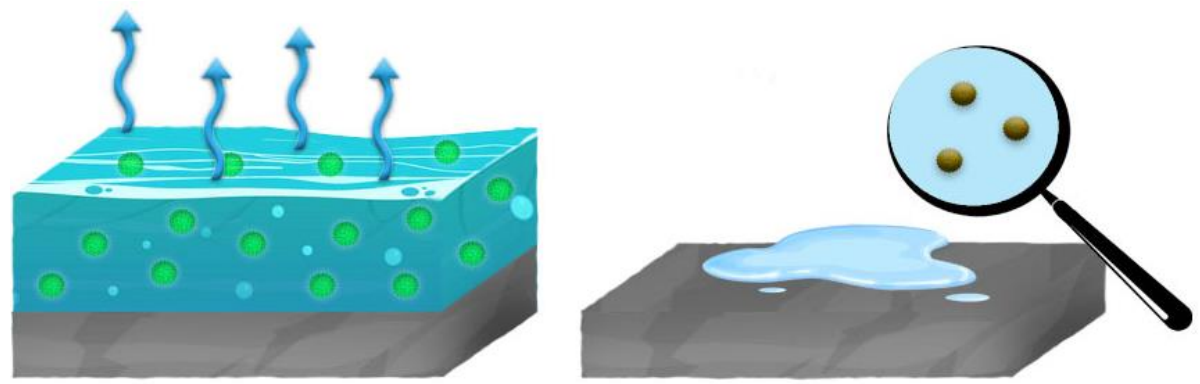

Fig. 1. The sketch for evaporation of a liquid sample with a viral culture from the solid surface.

Our experience in solving evaporation problems indicates that the evaporation rate of thin films and ensembles of aerosol particles depends on the thermal conductivity of the substrate (Khodyko et al., 2018). The evaporation proceeds faster from more heat-conducting materials and from thinner substrates. However, before quantifying and analysing the experimental data, two points have to be made. For the objectivity of the results we want to exclude copper and cardboard.

The highest death rate of the virus was observed on a copper substrate. It is known that copper and its compounds possess both antibacterial and antiviral activity (Noyce et al., 2007; Sunada et al., 2012). At the same time, such activity is absent on stainless steel substrate (Noyce et al., 2007). The evaporation of a liquid sample occurs most quickly also on copper as it is the most thermally conductive material. These facts lead to an assumption, that it can be the result of the occurrence of a synergetic effect, in which the antiviral properties of the copper (Sunada et al., 2012) are enhanced due to the effect of accelerated liquid evaporation discussed in this paper. There is some probability that the not only thermal conductivity and related liquid evaporation but different biological properties of the copper affect on the virus survival.

Regarding a cardboard, it should be noted that this is a porous material on which, in addition to evaporation, moisture is absorbed and diffused into the material. To clarify our analysis, we do not consider experimental data about an aerosol evaporation and virus survival on cardboard substrate. It is difficult to separate effects of evaporation and moisture absorption. Nevertheless, it is worthy to note that there are experimental data that respiratory viruses live less on absorbing surfaces (Tiwary et al, 2006). Therefore, only simulation results for stainless steel substrate and the plastic one can be correctly compared.

\section{MODEL AND METHODS}

We calculate the time of evaporation of the deposited liquid layer of thickness $h$ on the substrate of thickness $H$, taking into account the thermophysical properties of all the materials involved in the process (Fig. 1). This problem is governed by the one-dimensional non-stationary moving boundary problem known as the Stefan problem. Brownian diffusion of coronaviruses maintains their approximately uniform distribution in the sample volume, so the use of a onedimensional approach seems reasonable. For a substrate the temperature $T_{s}$ is governed by the heat conduction equation

$\rho_{s} c_{s} \frac{\partial T_{s}}{\partial t}=\lambda_{s} \frac{\partial^{2} T_{s}}{\partial z^{2}}$

and for the liquid layer evolution of the temperature $T_{\mathrm{w}}$ is governed by equation

$\rho_{w} c_{w} \frac{\partial T_{w}}{\partial t}=\lambda_{w} \frac{\partial^{2} T_{w}}{\partial z^{2}}$

subject to the isothermal initial conditions $\left.T_{s}(z)\right|_{t=0}=T_{0},\left.T_{w}(z)\right|_{t=0}=T_{0}$, and the second kind boundary condition at the boundary of the substrate and the liquid 
$\left.\lambda_{s} \frac{\partial T_{s}}{\partial z}\right|_{z=H}=\left.\lambda_{w} \frac{\partial T_{w}}{\partial z}\right|_{z=H}$

The location of the moving evaporating surface of the liquid is governed by the heat balance equation known as the Stefan condition

$\rho_{w}\left[c_{w} T_{w}(h(t))+U_{w}\right] \frac{d h}{d t}=-\lambda_{w} \frac{\partial T_{w}}{\partial z}+A_{w}+A_{a}$

here $\lambda_{s}, \lambda_{w}$ are the thermal conductivity coefficients of the substrate material and the evaporated liquid; $U_{w}$ is the specific heat of vaporization of the liquid; $\rho_{s}, \rho_{w}$ are, correspondingly, the mass density of the substrate and liquid; $A_{\mathrm{w}}$ and $A_{\mathrm{a}}$ are the energy flows carried by molecules of an evaporating liquid and a ballast gas, respectively. We suppose that evaporation is isothermal process, which is typical condition for the experiments described in discussed paper (Doremalen et al., 2020).

\section{RESULTS AND DISCUSSION}

Using the approach described in paper (Khodyko et al., 2018), from the solution of the Stefan problem (Eqs. (1)-(4)), for the time $\tau_{e v}$ of the complete evaporation of the liquid sample the following expression can be obtained

$\tau_{e v}=\frac{h \rho_{w} U_{w}}{2\left(T-T_{\text {lim }}\right)}\left(\frac{h}{\lambda_{w}}+\frac{H}{\lambda_{s}}\right)$

and the $T_{\text {lim }}$ is determined by the equation

$$
\rho_{s}\left(T_{\text {lim }}\right)=\rho_{s}(T) \varphi,
$$

where $\varphi$ is the relative humidity $(\mathrm{RH}), \rho_{\mathrm{s}}$ is mass density of saturated vapor.

To note that from experimentalist point of view $T_{\lim }$ is equal to the temperature of the wet thermometer which depends only on the temperature and humidity near the substrate. For experimental conditions (Doremalen et al., 2020) the relative humidity $\varphi$ is about of $40 \%$ and the difference $T-T_{\text {lim }}$ is equal to $14^{\circ} \mathrm{C}$. This temperature difference leads to the sample evaporation. It is worthy to emphasize that the temperature of the evaporation surface for quasi steady regime is approximately equal to $T_{\text {lim }}$.

For further calculations, we need to determine the thickness of the substrate and the evaporating film one. In the publication the thicknesses of the materials on which the viral substance was applied were not indicated (Doremalen et al., 2020). Therefore, from general considerations about the thicknesses of materials used in everyday life, we will take the thickness of the substrate equal to $H=1 \mathrm{~mm}$. This is, for example, the thickness of a credit card. The characteristic thickness of the liquid layer can be estimated only on the basis of the volume indicated in the article as $h \approx \mathrm{V}_{\mathrm{d}}^{1 / 3}=3.7 \mathrm{~mm}$. We assume that the evaporating liquid is close to some properties of water, for which $\lambda_{\mathrm{w}}=0.60 \mathrm{~W}(\mathrm{~m} \mathrm{~K})^{-1}, U_{\mathrm{w}}=2.26 \mathrm{MJ} \mathrm{kg}^{-1}$. Then for stainless steel substrate $\left(\lambda_{\mathrm{s}}=\right.$ $16.2 \mathrm{~W}\left(\mathrm{~m} \mathrm{~K}^{-1}\right)$ and polypropylene one $\left(\lambda_{\mathrm{s}}=0.22 \mathrm{~W}\left(\mathrm{~m} \mathrm{~K}^{-1}\right)\right.$, the evaporation time of a liquid layer with a thickness of $3.7 \mathrm{~mm}$ on plastic is equal to 3190 seconds, and on stainless steel is about 1850 seconds. In experiments, the lifetime of the virus on a plastic surface exceeds the lifetime on stainless steel substrate by approximately 1.7 times. Calculated, the ratio of evaporation times on different substrates is also 1.7 times. This proportion depends only on the physical properties of materials and their thicknesses. The absolute value of the evaporation time in this case also depends on relative humidity $(\mathrm{RH})$. Evaporation time increases significantly with increasing relative humidity because the value of $T_{\text {lim }}$ increasing also, see Exp. (5). No doubts that experiments with dangerous viral substances were carried out in closed volumes during several hours. Thus, liquid evaporation increases humidity in the experimental box. In our case an increase in the 
evaporation time by an order of magnitude can occur if the relative humidity rises from 40 to $85 \%$.

The primary experimental data (Doremalen et al., 2020) gives the dynamics of the change in the concentration of the virus over time. If our assumption is correct, then the death rate of viruses should strongly correlate with the evaporation rate of the liquid sample. Below we present the results of quantitative estimates that follow from this assumption. We then compare them with experimental data (Doremalen et al., 2020).

From the solution of the Stefan problem we can obtain the functional dependence of the height of the evaporating layer versus time for a substrate with poor thermal conductivity

$$
h=h_{0}-\frac{\lambda_{s}}{\rho_{w} U_{w}} \frac{\left(T-T_{\lim }\right)}{H} t=h_{0}-\beta t
$$

and for a substrate with high thermal conductivity

$$
h=\sqrt{h_{0}^{2}-\frac{\lambda_{w}}{\rho_{w} U_{w}}\left(T-T_{\lim }\right) t}=\sqrt{h_{0}^{2}-\alpha t}
$$

here $h_{0}$ is the initial layer height on the substrate.

For estimation of the dynamics in the concentration of viruses that are outside a living organism, we use the simplest Malthus model (Malthus et al., 1992). According to this model, the evolution of the concentration $C$ of viruses is described by the equation

$$
\frac{d C}{d t}=k C
$$

here $k$ is a parameter characterizing the dynamics of a population change. In the framework of the proposed approach, we believe that the parameter $k$ for the virus population will be proportional to the rate of evaporation $k=k^{\prime} V_{e v}=k^{\prime} d h / d t$. Then, for the two limiting cases of evaporation of a viral liquid sample, we have the following equations for the dynamics of the virus population. The equation for low heat conductive substrate $\left(\lambda_{s}<\lambda_{w}\right)$

$$
\frac{d C}{d t}=-k^{\prime} \frac{\lambda_{s}}{\rho_{w} U_{w}} \frac{\left(T-T_{\text {lim }}\right)}{H} C
$$

has solution

$$
\begin{aligned}
& C=C_{0} \exp \left[-\frac{t}{\tau_{l c}}\right] \\
& \tau_{l c}=\frac{\rho_{w} U_{w}}{k^{\prime} \lambda_{s}\left(T-T_{\text {lim }}\right)} H
\end{aligned}
$$

The equation for high heat conductive substrate $\left(\lambda_{\mathrm{s}} \gg \lambda_{\mathrm{w}}\right)$

$$
\frac{d C}{d t}=-\frac{k^{\prime} \alpha}{2 \sqrt{h_{0}^{2}-\alpha t}} C
$$

has solution

$$
C=C_{0} \exp \left[-k^{\prime} h_{0}\left(1-\sqrt{1-\frac{\alpha}{h_{0}^{2}} t}\right)\right]
$$


in the limit of short times, it follows from (13) that

$$
\begin{aligned}
& C \approx C_{0} \exp \left[-\frac{t}{\tau_{h c}}\right] \\
& \tau_{h c}=\frac{2 h_{0}}{k^{\prime} \alpha}=\frac{2 h_{0} \rho_{w} U_{w}}{k^{\prime} \lambda_{w}\left(T-T_{\text {lim }}\right)}
\end{aligned}
$$

here $\tau_{l c}$ and $\tau_{h c}$ are the characteristic lifetimes of viruses on a low heat-conducting and a high heat-conducting substrate respectively.

Our treatment of experimental data shown that there is explicit exponential decay of virus concentration over time (Fig. 2) (Doremalen et al., 2020). Moreover, for a stainless steel substrate, this characteristic time is $\tau_{\mathrm{hc}} \approx 7.4 \mathrm{~h}$, for a plastic substrate $\tau_{\mathrm{lc}} \approx 11.0 \mathrm{~h}$.

For a low heat-conducting substrate, the proposed model also predicts a simple exponential decay of the virus concentration over time. On a high conductive substrate, the concentration behavior is a more complex one. Asymptotically at initial times, virus concentration also has an exponential decay. But from Exp. (11) it follows that for this problem there is one more characteristic time $\tau_{\text {lim }}$

$$
\tau_{\lim }=\frac{h_{0}^{2}}{\alpha}=\frac{\rho_{w} U_{w} h_{0}^{2}}{\lambda_{w}\left(T-T_{\lim }\right)}
$$

This time is equal to twice the time of the liquid film evaporation in the considered approximation.

According to our model after time $\tau_{\text {lim }}$ the concentration of the virus becomes constant. It worthy to note that similar behaivior of virus concentration is observed in experimental data.

The obtained expressions allow us to draw several interesting conclusions. The large viral lifetimes observed in the discussed experiments are consistent with formulas (11) and (15) under

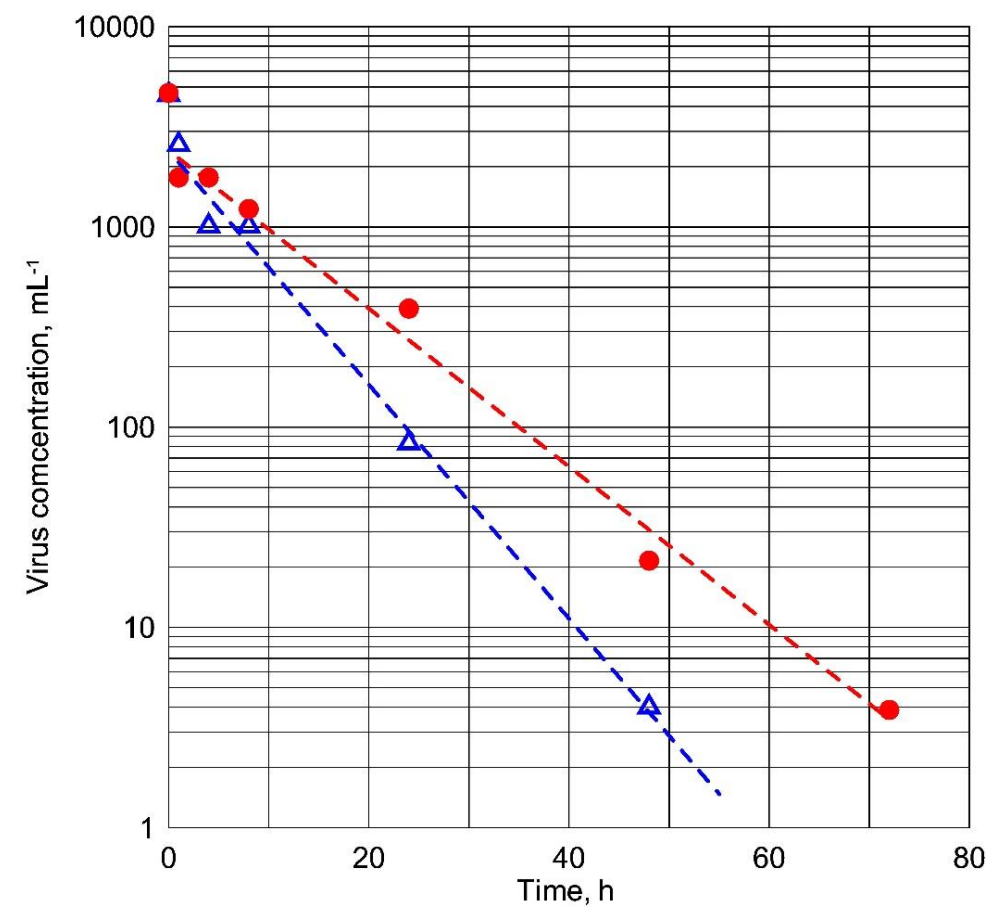

Fig. 2. Processing of experimental data (Doremalen et al., 2020) to determine the decay law in the concentration of SARS-CoV-2 virus. Triangles for stainless steel substrate; circles for plastic substrate. 
the condition of high humidity in the local environment of the substrate. An increase in air humidity will lead to an increase in the lifetime of the viral population in both cases. It follows from the expression (15) that the discussed lifetime for case of a high conductive substrate does not depend on the thickness of the substrate, but depends on the initial thickness of the liquid film. For a low heat-conducting substrate, it follows from the Exp. (11) that the lifetime of viruses in a liquid sample increases with a decrease in the thermal conductivity of the substrate and an increase in the total thickness of the substrate.

These conclusions require additional verification. Nevertheless they can play an important role for medical practitioners and researches.

Finally, let us estimate the rate of evaporation of an aerosol, which can be formed on various surfaces by sneezing or coughing, on a substrate.

Consider a practical example when, in isothermal conditions, an aqueous aerosol with a coronovirus is deposited on a solid surface. Using the approach related to the Stefan problem again (Khodyko et al., 2018), we obtain the estimation for the aerosol evaporation time $\tau_{a}$

$$
\tau_{a}=\frac{\rho_{w} U_{w} H R}{2 \lambda_{s}\left(T-T_{\lim }\right)}
$$

As it can be seen, the thermal conductivity of the substrate, the initial radius of the aerosol droplets $R$ (approximately the thickness of liquid layer), and the thickness of the substrate determine the time of the aerosol evaporation. During aerosol evaporation deformations near the evaporation front destroy viruses. It is known that during coughing the average droplet size of the aerosol is $5 \mu \mathrm{m}$ (Yang et al., 2007). While sneezing the aerosol has a generally bimodal distribution of drops with maxima in the size range of 100 and $600 \mu \mathrm{m}$ (Han et al., 2013). In Fig. 3 for two temperatures $22^{\circ} \mathrm{C}$ and $37^{\circ} \mathrm{C}$ the evaporation time of water aerosol particles with a diameter of 5 and $100 \mu \mathrm{m}$ are shown. The plastic substrate in these estimations has $2 \mathrm{~mm}$ thickness. Interestingly, that the human skin has thermal properties that are very similar to the plastic material ones (Hoque and Gandhi, 1988). Therefore, these calculations will be relevant for the estimation aerosol evaporation time on the surface of human skin. The evaporation time

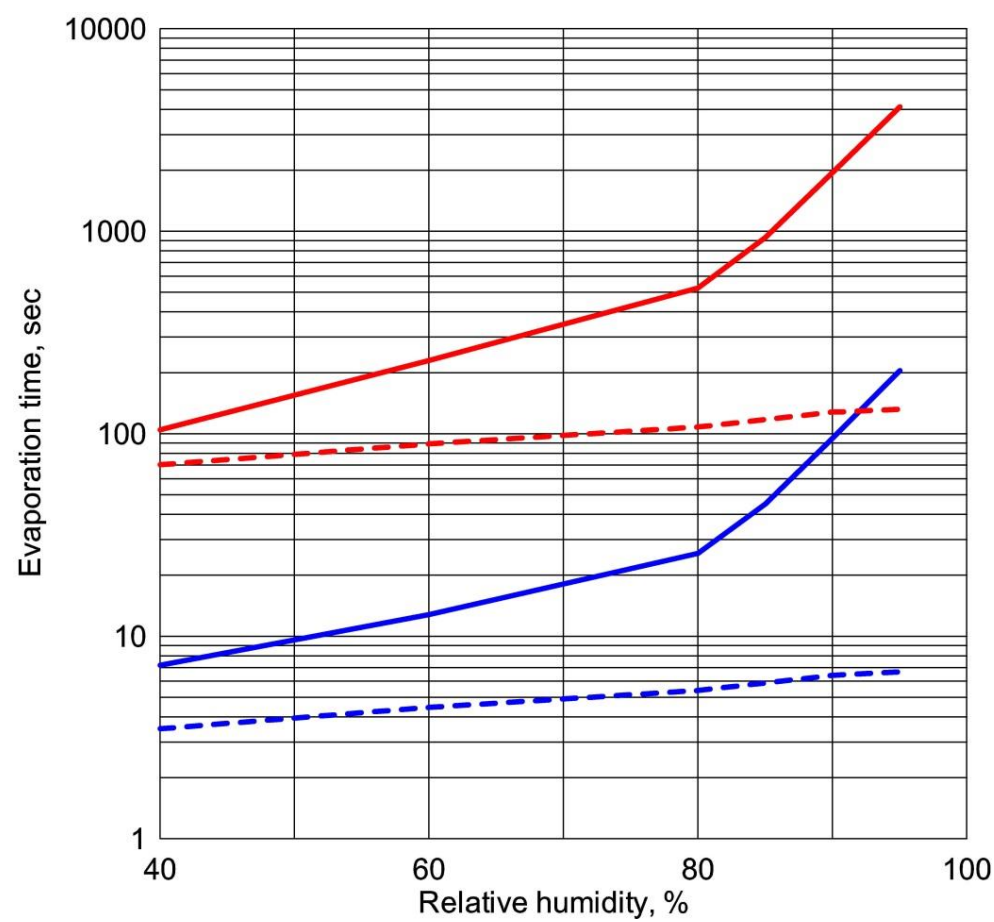

Fig. 3. Aerosol evaporation time on a $2 \mathrm{~mm}$ thick plastic substrate, depending on air humidity. Blue color is for $5 \mu \mathrm{m}$ aerosol droplets, red color is for $100 \mu \mathrm{m}$ droplets. Solid lines are for substrate and air temperature $22^{\circ} \mathrm{C}$; dotted lines are for substrate temperature $37^{\circ} \mathrm{C}$, air temperature $22^{\circ} \mathrm{C}$. 
range varies widely and is sensitive to air humidity near the substrate. At $100 \%$ relative humidity and isothermal condition, the geometrical sizes of any aerosol do not change at all.

\section{CONCLUSIONS}

The physical model is proposed for vitality of aqueous samples with coronavirus on different substrates. It is shown that the evaporation rate of a liquid sample containing the culture of coronavirus affects its survival on a substrate. Possible mechanisms of such influence can be due to the appearance of large non-comprehensive capillary pressures about 140 bars and the associated dynamic forces during the movement of the evaporation front in a droplet sample with the virus. A simulation of isothermal evaporation of a thin liquid sample based on the Stefan problem was performed. It was shown that evaporation time of the aqueous sample is inversely proportional to the thermal conductivity of the substrate $\lambda_{\mathrm{s}}$ and directly proportional to the substrate thickness $H$. The evaporation time is directly proportional to the sample radius. Therefore, the shortest evaporation time simulation shown is for aqueous sample on the cupper substrate. We did comparison with experimental data about evaporation droplets with radii about $3.7 \mathrm{~mm}$. The presented theory cannot cover all physical aspects of the complex problem of the vital functions of viruses in aqueous samples. Nevertheless, we believe that it revealed an important connection between the rate of evaporation of liquid biological samples and the vitality of coronavirus. It was shown that the main experimental results on the dynamics of coronavirus survival on various surfaces (Doremalen et al., 2020) correlate well with the rate of isothermal evaporation of aqueous samples with viruses on substrates which do not absorb moisture. The evaporation rate depends on the thermal conductivity and thickness of the substrate, as well as the relative humidity of the air. Ceteris paribus, the viral culture should die faster on more thermally conductive and thinner substrates, as well as in lower relative humidity environment, which provide a higher evaporation rate.

\section{ACKNOWLEDGEMENTS}

This work was supported by the Belarussian Republican Foundation for Fundamental Research. The authors wish to thank Professor Oleg Penyazkov for the support of this work.

\section{DISCLAIMER}

The authors declare that they have no conflicts of interest.

\section{REFERENCES}

Aggarwal, A. (2018). Determination of prestress and elastic properties of virus capsids. Phys. Rev. E 97, 032414. https://doi.org/10.1103/PhysRevE.97.032414

Alvarez-Zuzek, L.G., Di Muro, M.A., Havlin, S., Braunstein L.A. (2019). Dynamic vaccination in partially overlapped multiplex network. Phys. Rev. E 99, 012302. https://doi.org/10.1103/Phys RevE.99.012302

Boulos, M.N.K., Geraghty, E.M. (2020). Geographical tracking and mapping of coronavirus disease COVID-19/severe acute respiratory syndrome coronavirus 2 (SARS-CoV-2) epidemic and associated events around the world: How $21^{\text {st }}$ century GIS technologies are supporting the global fight against outbreaks and epidemics. Int. J. Health Geographics 19, 8. https://doi.org/1 0.1186/s12942-020-00202-8

Cui, J., Li, F., Shi, Z.L. (2019). Origin and evolution of pathogenic coronaviruses. Nat. Rev. Microbiol. 17, 181-192. https://doi.org/10.1038/s41579-018-0118-9

Frank, I.W., Tanenbaum, D.M., van der Zande, A.M., McEuen P.L. (2007). Mechanical properties of suspended graphene sheets. J. Vac. Sci. Technol., B 25, 2558. https://doi.org/10.1116/1.278 9446

Han, Z.Y., Weng, W.G., Huang, Q.Y. (2013). Characterizations of particle size distribution of the 
droplets exhaled by sneeze. J. R. Soc. Interface 10, 20130560. https://doi.org/10.1098/rsif.201 3.0560

Hoque, M., Gandhi, O.P. (1988). Temperature distributions in the human leg for VLF-VHF exposures at the ANSI-recommended safety levels. IEEE Trans. Biomed. Eng. 35, 442. https://doi.org/10.1109/10.2114

Khodyko, Y.A., Saverchenko, V.I., Fisenko, S.P. (2018). Features of evaporation of an ensemble of femto-picoliter droplets on a substrate. Experiment and simulation. Interfacial Phenom. Heat Transfer 6, 231. https://doi.org/10.1615/InterfacPhenomHeatTransfer.2019029624

Lee, C., Wei, X., Kysar, J.W., Hone, J. (2008). Measurement of the elastic properties and intrinsic strength of monolayer graphene. Science 321, 385-388. https://doi.org/10.1126/science.1157 996

Li, X., Geng, M., Peng, Y., Meng, L., Lu, S. (2020). Molecular immune pathogenesis and diagnosis of COVID-19. J. Pharm. Anal. 10, 102-108. https://doi.org/10.1016/j.jpha.2020.03.001

Malthus, T.R., Winch, D., James P. (1992). An essay on the principle of population. Cambridge University Press.

Noyce, J.O., Michels, H., Keevil, C.W. (2007). Inactivation of influenza A virus on copper versus stainless steel surfaces. Appl. Environ. Microbiol. 73, 2748. https://doi.org/10.1128/AEM.0113906

Shaman, J., Kohn, M. (2009). Absolute humidity modulates influenza survival, transmission, and seasonality. Proc. Natl. Acad. Sci. U.S.A. 106, 3243. https://doi.org/10.1073/pnas.0806852106

Sunada, K., Minoshima, M., Hashimoto, K. (2012). Highly efficient antiviral and antibacterial activities of solid-state cuprous compounds. J. Hazard. Mater. 235, 265. https://doi.org/10.101 6/j.jhazmat.2012.07.052

Tiwari, A., Patnayak, D.P., Chander, Y., Parsad, M., Goyal, S.M. (2006). Survival of two avian respiratory viruses on porous and nonporous surfaces. Avian Dis. 50, 284. https://doi.org/10.1 637/7453-101205R.1

van Doremalen, N., Morris, D.H., Holbrook, M.G., Gamble, A., Williamson, B.N., Tamin, A., Harcourt, J.L., Thornburg, N.J., Gerber, S.I., Lloyd-Smith, J.O., de Wit, E., Munster V.J. (2020). Aerosol and surface stability of SARS-CoV-2 as compared with SARS-CoV-1. N. Engl. J. Med. 382, 564-1567. https://doi.org/10.1056/NEJMc2004973

Wang, W.N., Jiang, Y. and Biswas, P. (2012). Evaporation induced crumpling of graphene oxide nanosheets in aerosolized droplets: Confinement force relationship. J. Phys. Chem. Lett. 3, 3228-3233. https://doi.org/10.1021/jz3015869

Wu, C., Liu, Y., Yang, Y., Zhang, P., Zhong, W., Wang, Y., Wang, Q., Xu, Y., Li, M., Li, X., Zheng, M., Chen, L., Li, H. (2020). Analysis of therapeutic targets for SARS-CoV-2 and discovery of potential drugs by computational methods. Acta Pharm. Sin. B 10, 766-788. https://doi.org/10.1016/j.a psb.2020.02.008

Yang, S., Lee, G.W.M., Chen, C.M., Wu, C.C., Yu, K.P. (2007). The size and concentration of droplets generated by coughing in human subjects. J. Aerosol Med. 20, 484. https://doi.org/1 0.1089/jam.2007.0610

Yang, W., Marr, L.C. (2012). Mechanisms by which ambient humidity may affect viruses in aerosols. Appl. Environ. Microbiol. 78, 6781-6788. https://doi.org/10.1128/AEM.01658-12

Zandi, R., Reguera, D. (2005). Mechanical properties of viral capsids. Phys. Rev. E 72, 021917. https://doi.org/10.1103/PhysRevE.72.021917 